\title{
Transient Bioelectronics: Electronic Properties of Silver Microparticle- Based Circuits on Polymeric Substrates Subjected to Mechanical Load
}

\author{
Reihaneh Jamshidi, Simge Çinar, Yuanfen Chen, Nastaran Hashemi, Reza Montazami
}

Department of Mechanical Engineering, lowa State University, Ames, lowa, 50011

Correspondence to: R. Montazami; (E-mail: reza@iastate.edu)

published online 00 Month 2015

DOI: 10.1002/polb.23804

ABSTRACT: Transient soft bioelectronics are capable of forming conformal contacts with curvilinear surfaces of biological host tissues and organs. Such systems are often subject to continuous static and dynamic loads from the biological host. In this article, we present investigation of electronic attributes of transient soft bioelectronic circuits subjected to mechanical force and influence of substrate's transiency on the transiency of the whole device; also, characterize and quantify loss of functionality in triggered devices. Variations in the electrical conductivity of circuits as a function of applied mechanical load was used as a means to deduce electronic characteristics under stress. The experimental results suggest that there exists a correlation between electronic properties of circuits and applied mechani- cal strain; no clear correlation was, however, observed between electronic properties of circuits and frequency of the applied dynamic load. Control over transiency rate of identical circuits utilizing the transiency characteristics of the poly(vinyl alcohol)l-based substrates is also studied and demonstrated. (C) 2015 Wiley Periodicals, Inc. J. Polym. Sci., Part B: Polym. Phys. 2015, 00, 000-000

KEYWORDS: bioelectronics; biomaterials; degradation; functional polymers; mechanics of soft electronics; structureproperty relations biodegradable; transient electronics; transient polymers
INTRODUCTION Recent advances on integration of electric circuits on advanced soft materials have enabled an emerging field at the junction of materials science, electronics, and biology. Bioelectronics, due to their unique characteristics, such as biocompatibility and flexibility, allow direct integration of electronic devices on curvilinear biological tissues. ${ }^{1-5}$ Thus far, there has been significant improvement on active (e.g., pacemakers), and passive (e.g., artificial hips and joints) nondegradable biomedical components, as well as passive (e.g., sutures) bioresorbable biomedical components. However, active bioresorbable components (bioelectronics) is still an immature field of study with great potentials and opportunities. Recently, integration of biodegradable organic and inorganic electronics on transient substrates has enabled the development of transient bioelectronics. Transient electronics, in general, is an emerging field in materials science and engineering focused on materials and structures that can maintain full functionality up to the point they are no longer needed, and undergo fast self-destruction when transiency is triggered. Trigger and triggering mechanisms vary from heat/melting ${ }^{6}$ to solvent/dissolution ${ }^{7,8}$ to light/photodecomposition, ${ }^{9}$ to name few examples. Transient bioelectronics with controlled transiency rate are realized by fabrication of circuits and electronic components of organic and inorganic biodegradable materials ${ }^{8,10-13}$ on substrates with controlled transiency rate ${ }^{14,15}$; and can limit or completely eliminate the need for secondary invasive surgeries required to remove implanted bioelectronics, once the operation period is completed. $^{7}$ Controlling transiency rate of electronic components of transient bioelectronics is very often more challenging than that of transient substrates because the electronic properties of electronic components may be compromised for transiency attributes, which is overall undesirable.

Early works on transient materials and electronics started with electronics on bioresorbable substrates and also partially transient electronics with certain degradable components. Instances of the first studies include conformal bioelectronics based on bioresorbable silk substrate in which dissolution of silk initiates wrapping of electronics around biological tissues. ${ }^{16,17}$ Instances of partially dissolvable electronics include devices in which all components except electrodes and interconnects are dissolvable in biological medium. ${ }^{12,15}$ Recently, fully dissolvable electronics have been reported by Rogers et al. They use silicon as the semiconductor, metal oxides $\left(\mathrm{MgO}, \mathrm{SiO}_{2}\right)$ as dielectrics, $\mathrm{Mg}$ for conductors and silk as the substrate and packaging material. ${ }^{7,8}$ Another example of a fully transient electronic is a RF pressure sensor which uses a $\mathrm{Zn} / \mathrm{Fe}$ bilayer as the sensor conductor material, poly-L-lactide as dielectric and polycaprolactone as the structural material. ${ }^{18}$ More recently high performance transient electronics, ${ }^{11}$ 

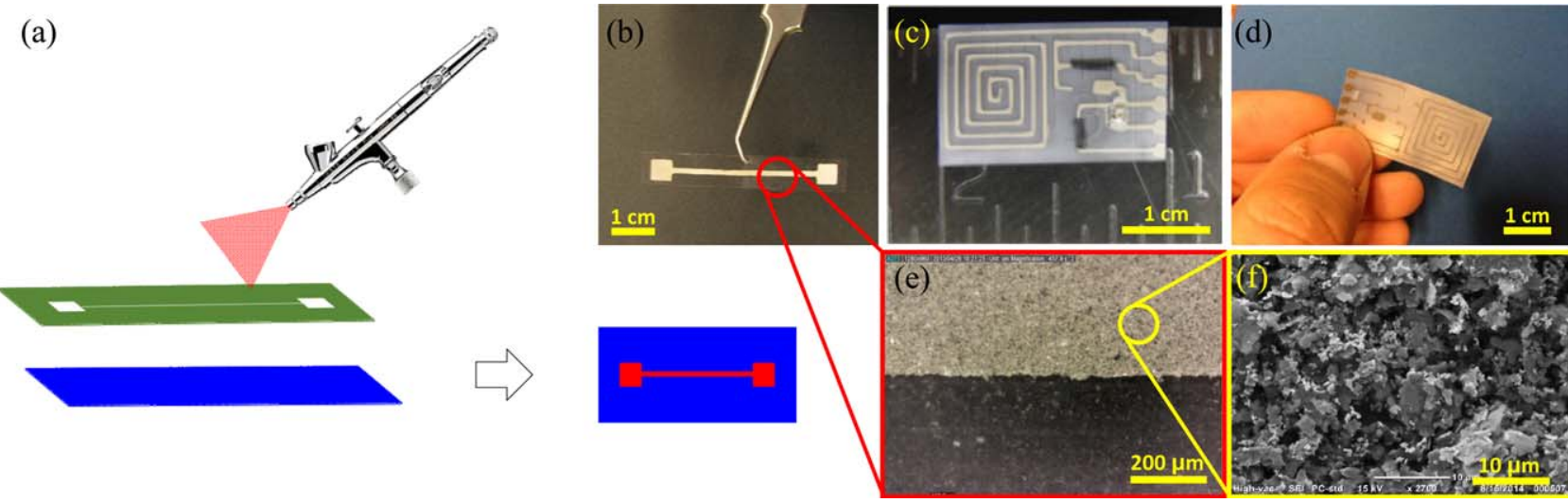

FIGURE 1 Patterning PVA-based substrates with silver ink. (a) diluted silver ink is spray-coated on the substrate over a patterned mask; (b) a basic circuit consisting of a straight conductive path fabricated on PVA-based substrate, similar samples were used for electrical-mechanical testing when resistance was monitored; (c and d) a receiver circuit consisting of an antenna and capacitor fabricated from silver ink and carbon-based resistors on PVA substrate, similar samples were used to receive wirelessly transmitted signals; (e) optical microscopy image of a silver ink conductive path (light) on PVA substrate (dark); (f) SEM image of silver ink coated electrode. [Color figure can be viewed in the online issue, which is available at wileyonlinelibrary.com.]

transient batteries, ${ }^{19}$ and programmable transient electronics $^{20}$ are also reported.

Early studies toward realization of transient bioelectronics initiated and evolved around addressing the technological gap in active bioresorbable biomedical devices by fabrication of biocompatible transient electronic components. Basic transient bioelectronics have been fabricated by patterning inorganic and organic conductive materials on a variety of soft transient substrates of natural and synthetic materials. To our best knowledge, the first reported transient electronics component was a field-effect transistor based on poly(vinyl alcohol) (PVA) as gate dielectric, and 5,5'-Bis(7-dodecyl-9Hfluoren-2-yl)-2,2'-bithiophene (DDFTTF) as organic semiconductor on poly(L-lactide-co-glycolide) (PLGA) substrate; reported by Bettinger et al. More recently, studies have been reported on wide range of organic materials for bioelectronics. $^{21-23}$

Transiency rate and correlation between mechanical force and electrical properties are critical to success of transient soft bioelectronics. Due to their low elastic modulus, soft electronics may undergo large strain when subjected to even small magnitudes of stress; such deformation may influence and alter the electronic characteristics of the electronic components. In our previous studies we have investigated soft ionic polymers for their electronic and mechanical properties in form of soft actuators and sensors, ${ }^{24-35}$ and in healthcare applications ${ }^{36,37}$ also, we have investigated use of PVA-based polymer composites to control transiency rate. ${ }^{14}$ In this work, we have fabricated a series of prototypical circuits based on spray-coated silver microparticle conductive paths on PVA-based substrates (Fig. 1) and have investigated their environmental stability as well as strain-dependence of their electronic properties to establish a correlation between applied stress and electronic properties in transient soft bio- electronics. We have also studied transiency behavior of these circuits fabricated on different PVA-based substrates to understand the correlation between the transiency rate of substrate and that of the whole system. We utilized complex circuits [Fig. 1(c,d)] to demonstrate signal transition to a transient flexible electronic device and characterized transiency as a function of signal strength. Finally, we demonstrated that the transiency rate of the membrane can be utilized as a means to control and program that of the bioelectronics.

\section{RESULTS AND DISCUSSION}

\section{Environmental Stability}

Maintenance of electrical and mechanical properties under ambient conditions is highly desired for bioelectronic devices as it guarantees sufficient functionality when transiency is not yet triggered. In our previous work, ${ }^{14}$ we have investigated the mechanical properties of transient PVA substrates with controlled transiency rate as a function of the environmental conditions. As part of the present study we have investigated the electrical stability of circuits on transient PVA substrates for maintaining their electrical properties when exposed to the ambient conditions (average relative humidity of $75 \%$ at $22{ }^{\circ} \mathrm{C}$ ) over a period of several days. Presented in Figure 2 is the measured resistance of an arbitrary electronic circuit [similar to that shown in Fig. 1(b), 5 $\times 5 \mathrm{~mm}^{2}$ connection points connected by a $33 \times 1.5 \mathrm{~mm}^{2}$ path] fabricated via spray coating on a pure PVA substrate over a 4-day period. The resistance is unchanged and stable to micro-ohm scale from the first hours (inset) to the end of the 4-day data collection period. Results suggest that, although it is unpackaged and fully exposed to ambient conditions, the circuit and the substrate completely maintain their mechanical and electrical integrity and do not exhibit 


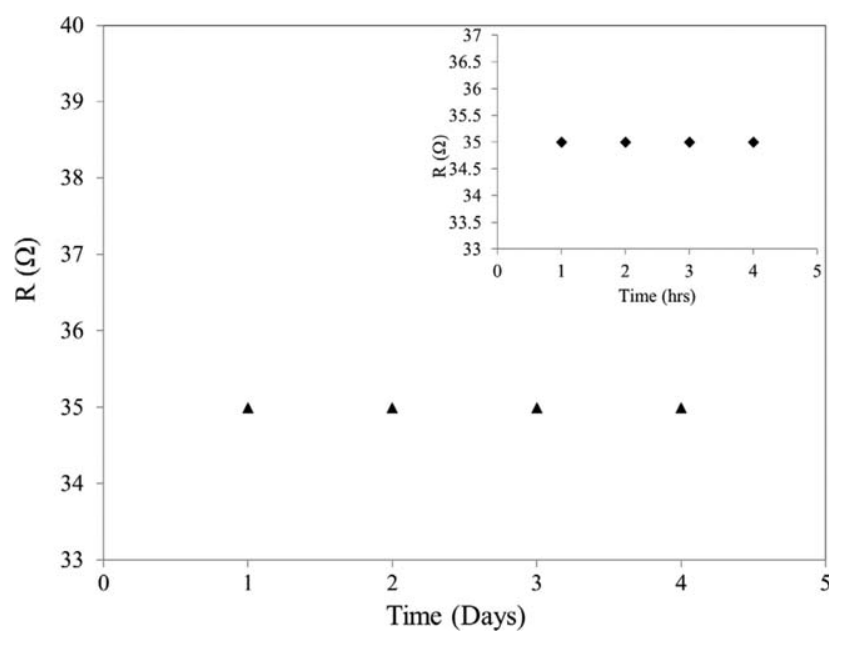

FIGURE 2 Electrical stability of PVA-silver circuit in ambient conditions over a 4-day period. Inset represents data for the first $4 \mathrm{~h}$.

any loss of functionality. This is particularly important as even a slight change in electrical characteristics of electronic components may result in loss of functionality of the whole electronic circuit. Transient bioelectronics, just like ordinary electronics, must maintain their mechanical and electrical stability during their operational period, and be capable of rapidly undergoing loss of functionality and degradation once transiency is triggered.

\section{Strain-Electronic Properties Correlations}

Epidermal and implantable bioelectronics, depending on their host organ, have high probability of being subjected to mechanical stress from the host biological tissue. For instance, epidermal bioelectronics are subject to tension and compression exerted by the skin; and, if implanted on or in close vicinity of a muscle, subject to dynamic stress due to contraction and relaxation of the muscle. It is important to establish correlations among the applied stress, strain and electronic characteristics of bioelectronics to understand and compensate for potential fluctuations in the overall electronic characteristics of bioelectronics. We have examined electrical properties of transient circuits fabricated on PVA substrates in response to both static and dynamic loads. For purpose of discussion, electronic resistance is used as a means to deduce variations in the electronic properties of the circuits.

\section{Static Load}

Resistance of a circuit similar to that presented in Figure 1 (b) was investigated in response to strain due to a force of 0-10 $\mathrm{N}$ increasing at a step size of $0.5 \mathrm{~N} / \mathrm{min}$, load condition at each step was treated as static. As presented in Figure 3, the resistance was measured and monitored as a function of strain and exhibited an increasing trend with increasing strain. Resistance remained approximately unchanged for up to $0.15 \%$ strain; and increased uniformly subsequently up to approximately $1.3 \%$, where the resistance reached a plateau followed by nonuniform fluctuations which are most prob- ably indications of failure and significant fracture of the silver coating.

The conductive path is deposited from silver microparticles [Fig. 4(a)] thus it does not behave elastically once elongated. The increase in resistance is mainly due to partial separation of the silver particles and microcrack formation in the conductive path [Fig. 4(b)] that retards current flow; however, elasticity of the PVA substrate could bring the silver microparticles back together and fully or partially recover the conductivity when the stress is removed [Fig. 4(c,d)]. This is evident in the data presented and discussed in the subsequent section, Dynamic Load.

\section{Dynamic Load}

Electronic properties dependence on dynamic load, which is a more realistic comparison to a biological host tissue, was carried at different frequencies and displacements. Presented in Figure 5 are fluctuations in the resistance of a circuit subjected to $180 \mu \mathrm{m}$ displacement (corresponds to a strain of $1.8 \%$ ) at $0.02 \mathrm{~Hz}$ frequency. The fluctuations are almost fully reversible, although a lag is observed when the stress is removed, which is due to the viscoelastic behavior of the PVA substrate. After removal of the stress at midpoint of each cycle, the PVA substrate recovers from the elongated state back to the neutral state; this recovery, however, is not immediate. As evident from the data, a large portion of recovery $(60-70 \%)$ occurs instantaneously and the remaining occurs over a period of time.

Overall behavior at higher frequency $(1 \mathrm{~Hz})$ and different (12 and $180 \mu \mathrm{m}$ ) displacements (corresponding to strains of 0.12 and $1.8 \%$, respectively) was very similar to that at lower frequency. As shown in Figure 6, a correlation exists between strain and resistance; a clear correlation between frequency and resistance, however, is not evident from our experiments. For high frequency testing, the sample was subjected to cyclic low and high strains; a lag was observed in low-strain data at high frequency testing; that is, the

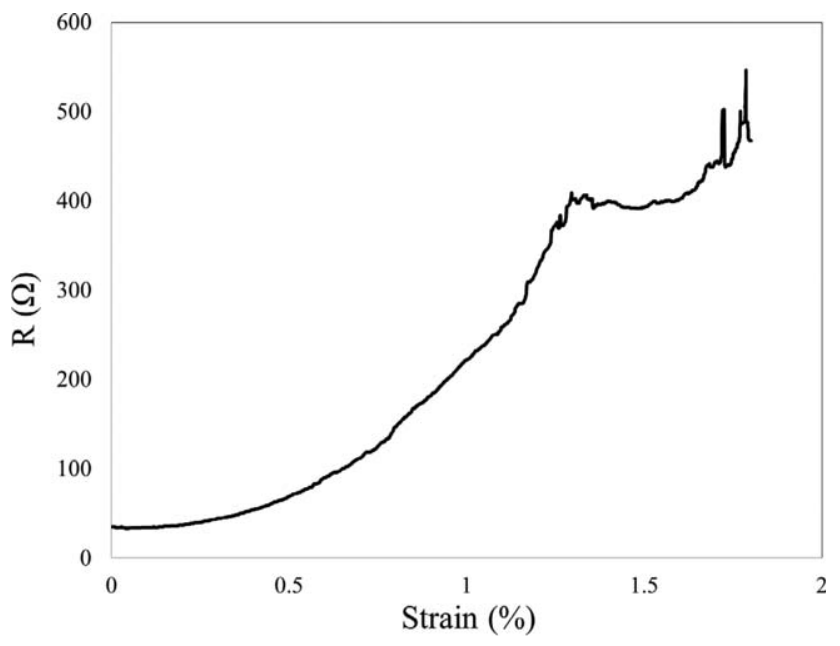

FIGURE 3 Resistance of silver microparticle conductive path is directly proportional to the elongation of circuit under load. 

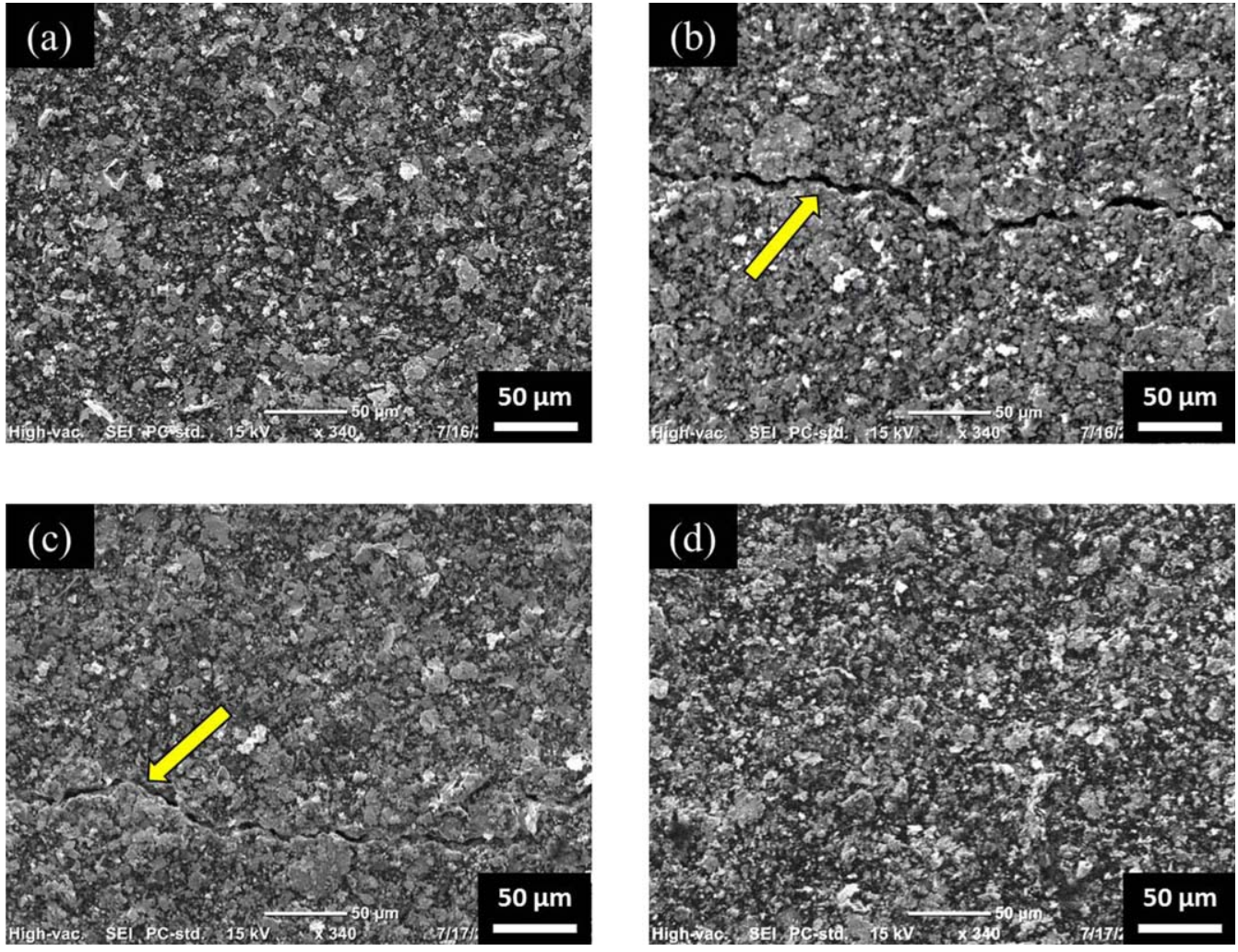

FIGURE 4 SEM micrographs of (a) silver ink coating on PVA substrate; (b) under $1.8 \%$ strain; (c) after strain is removed with visible partially recovered fracture; and d) an intact area after strain is removed. Yellow arrows point at a fracture point while sample is strained (b) and a partially recovered fracture after strain is removed (c). [Color figure can be viewed in the online issue, which is available at wileyonlinelibrary.com.]

resistance peak in low-strain data occurs slightly after that of the high-strain data. The second peak on each cycle corresponds to the period of time when the stress is evidently removed; however, despite initial drop of resistance reading, it does slightly increase before decreasing again to the neutral (initial value). The nature of the lag and secondary peaks

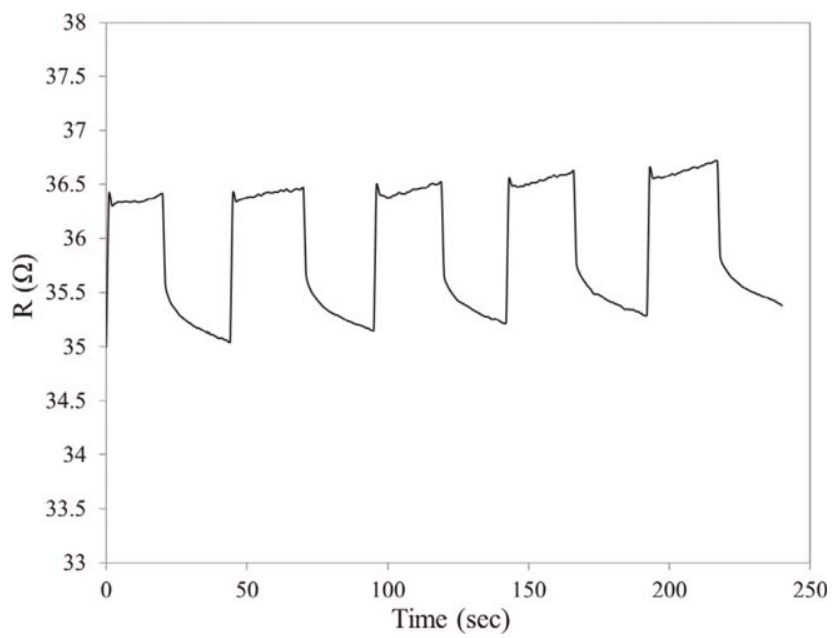

FIGURE 5 Variations in electric resistance of a silver microparticle conductive path subjected to $1.8 \%$ strain at frequency of $0.02 \mathrm{~Hz}$. are still unknown to us. They may be due to equipment limitations and/or nonlinear behavior of the substrate or circuit.

Also, over numerous cycles an overall increase in resistance was observed. Increase in overall resistance, although very small and detectable only after tens of cycles (Fig. 7), may

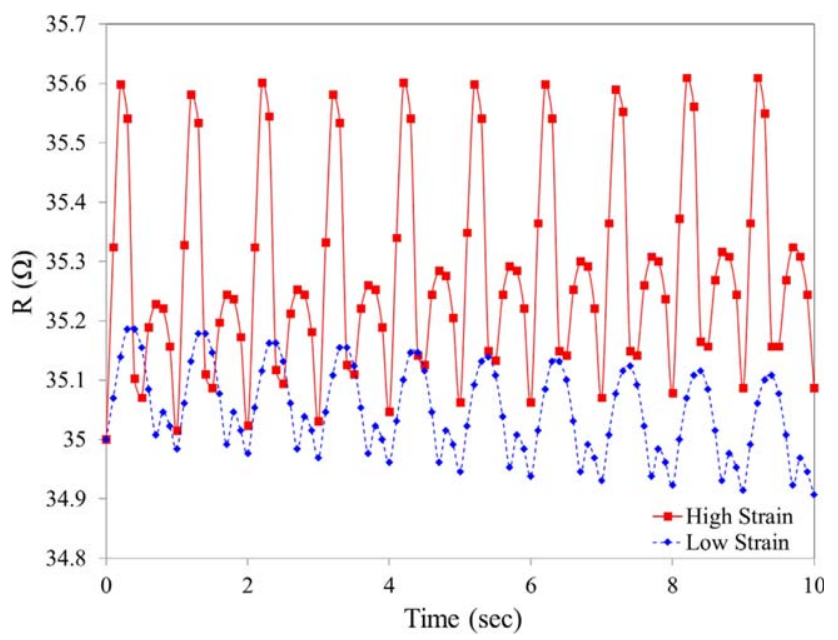

FIGURE 6 Electrical response of a straight conductive path as a function of low and high strain at frequency of $1 \mathrm{~Hz}$. [Color figure can be viewed in the online issue, which is available at wileyonlinelibrary.com.] 


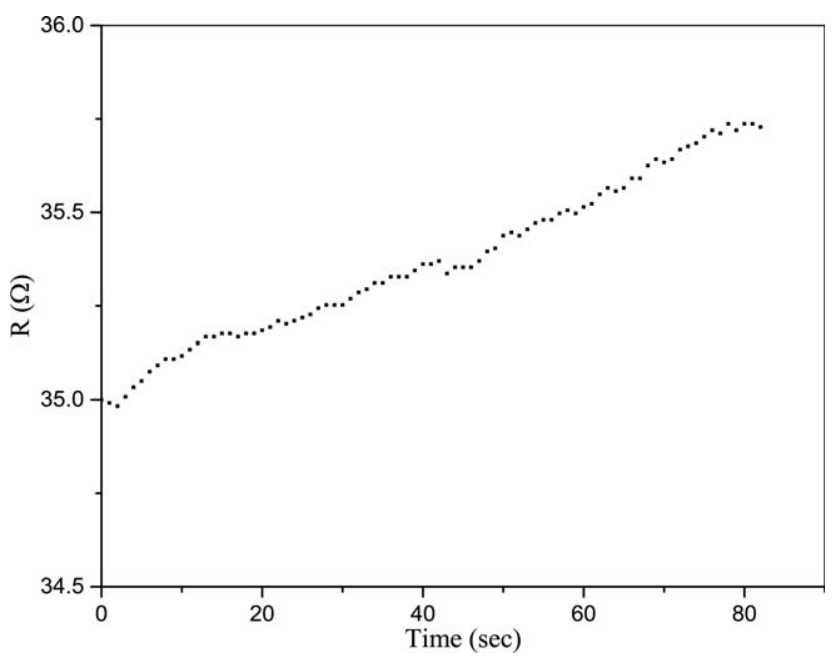

FIGURE 7 The overall resistance increases after numerous cycles of $1.8 \%$ strain.

be an indication of change in electronic properties in longterm; a longer relaxation period may be needed to fully recover from this state, such condition (tens of consecutive cycles) is rare in biological systems except for few organs like heart.

What makes soft and flexible electronics vulnerable to malfunction is variations in electronic properties when subjected to strain. Elastic modulus of soft electronics is orders of magnitude less than that of ordinary silicon-based electronics; thus, even small forces can cause significant deformation and elongation in soft electronics that will affect their electronic properties. Our studies presented in this paper suggest existence of a reversible correlation between electronic properties and strain; yet, non between electronic properties and frequency of the applied stress. This observation is mainly due to the mismatch of elastic moduli between polymeric substrates and electronic components. Under larger elongation of PVA substrate, silver microparticles are distanced from each other, which causes a drop in conductivity; upon removal of force, the elasticity of polymeric membrane brings the electronic components back together and results in the recovery of electrical conductivity. At higher frequency (1 Hz) a similar behavior was observed. Viscoelasticity of polymeric membrane resulted in a lag in recovering from the stress after it was removed. Considering significant variations in electrical resistance as a function of strain, development of conductive material with elastic moduli closer to that of soft polymers appears to be necessary for fabrication of, especially large area, soft electronics, and bioelectronics.

\section{Transiency}

Simple Circuit: Circuits consisting of single electrodes [Fig. 1(b)] were fabricated on PVA-sucrose substrates of different content ratios. The experiment was aimed to identify the role of substrates' transiency on defining the transiency rate of the whole circuit. Similar substrates without circuits were previously studied in our group and it was observed that addition of sucrose to PVA matrix, beyond 2:1 (PVA:sucrose) ratio (corresponds to $0.5 \mathrm{~S}$ sample) will expedite the transiency. ${ }^{14}$ In this study, identical conductive patterns were fabricated on pure (10:0), $0.1 \mathrm{~S}(10: 1), 0.5 \mathrm{~S}$ (10:5), and $2 \mathrm{~S}$ (10:20) PVA-sucrose membranes and resistance was monitored and recorded as a function of exposure time to DI water. The experimental data suggested that transiency rate increases with increasing concentration of sucrose. The $2 \mathrm{~S}$ circuit, having the highest sucrose ratio undergo most significant loss of functionality and the sample degraded very rapidly resulting in a significant drop of electrical conductivity. Since the transiency of membrane is deriving that of the whole circuit, this information is particularly important in design of bioelectronic devices with controlled transiency rate. Presented in Figure 8 are resistance curves for samples of different composition over 1100 s. Pure PVA sample and those with lower concentrations of sucrose degrade more uniformly as their composition is more homogeneous. In samples with higher sucrose concentration, like 2S, sucrose crystals are formed which result in a phase-separated composite. These sucrose crystals can undergo very quick dissolution and result in fast degradation of the whole system and thus drop of electrical conductivity. Formation of sucrose crystals and their influence on transiency rate of polymer substrates is discussed in our previous study. ${ }^{14}$

\section{Complex Circuit}

A receiver circuit [Fig. 1(c)] was designed and fabricate using silver ink and carbon on PVA substrate. The circuit was designed to receive a signal sent by an inducting coil over a short distance (approximately $50 \mathrm{~cm}$ ). The receiver was exposed to DI water as the received signal was monitored. Unlike the previous study (demonstrated in Fig. 8 in which loss of electronic conductivity was interpreted as loss of functionality in electronic circuits, here loss of the received signal was used to quantify loss of functionality and

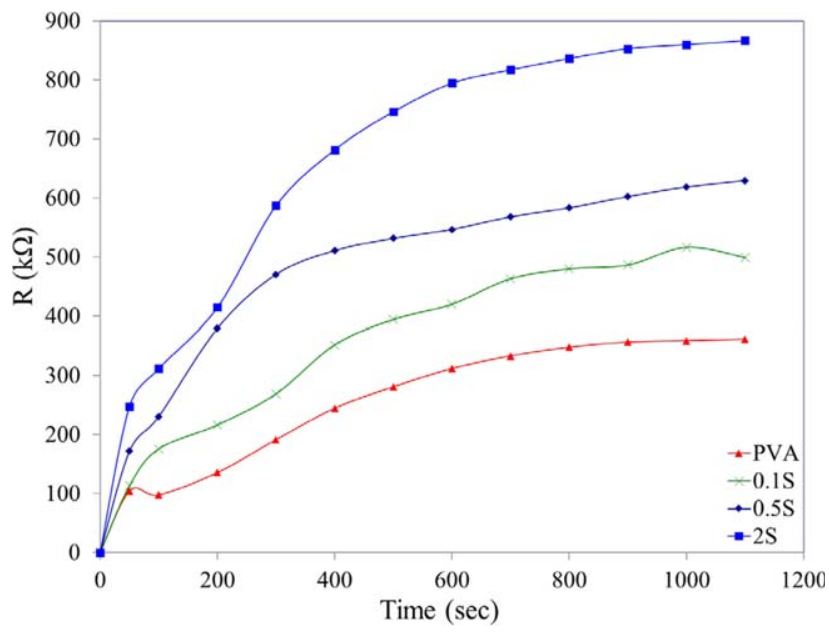

FIGURE 8 Transiency of a straight conductive paths fabricated on different substrates are quantified from loss of electrical conductivity when exposed to DI water. [Color figure can be viewed in the online issue, which is available at wileyonlinelibrary.com.] 

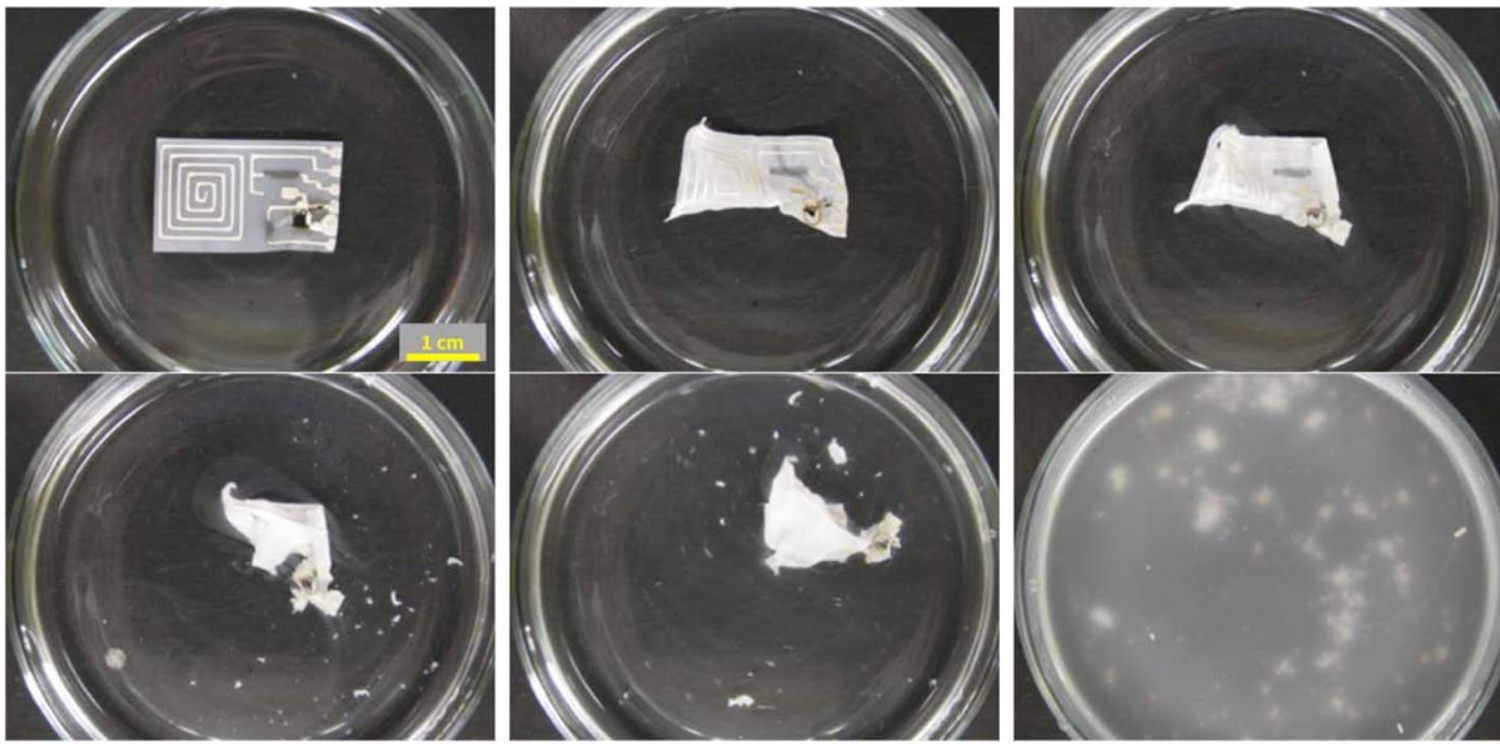

FIGURE 9 Sequential images of transiency of a circuit designed to receive signal from a nearby inducting coil. The images are recorded over a period of $500 \mathrm{~s}$. [Color figure can be viewed in the online issue, which is available at wileyonlinelibrary.com.]

transiency of the device. Sequential images in Figure 9 show a receiver circuit as it undergoes transiency over period of $500 \mathrm{~s}$. The last image (bottom-right) is after a slight agitation of the petri dish.

Signal from the receiver was monitored and recorded using an oscilloscope over a period of $450 \mathrm{~s}$; beyond this duration the connections were all degraded, which made it impossible to collect reliable data. The collected data are shown in Figure 10 .

This experiment demonstrates and confirms wireless transmittance of signal to a transient device as it is undergoing

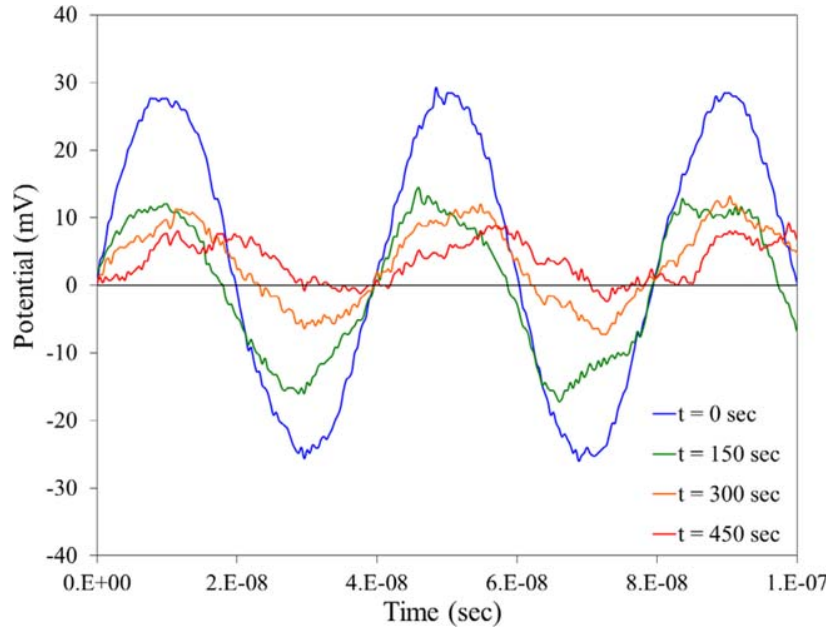

FIGURE 10 Overlapped signals received by the receiver circuit and recorded by an oscilloscope. The sample losses functionality over a period of approximately $450 \mathrm{~s}$. [Color figure can be viewed in the online issue, which is available at wileyonlinelibrary.com.] transiency. Transiency of the polymer substrate plays an important role in breaking up the circuit and contributes to expedited transiency rate. As the receiver is degrading, in addition to amplitude of peaks, their location is also changing and shifting to the right, indicating that the device is losing its intended functionality. The baseline signal (picked up by the clamps and cables before connecting the receiver) is subtracted from the presented data. Positive peaks of the received signals are plotted in Figure 11, showing loss of functionality of the electronic device as a function of time.

Transiency experiments suggest that transiency rate of PVAbased substrate is dominating the mechanism of degradation and transiency of the whole device. Degradation and transiency rates of the bioelectronic devices can be controlled and tuned by controlling transiency of the polymeric substrate

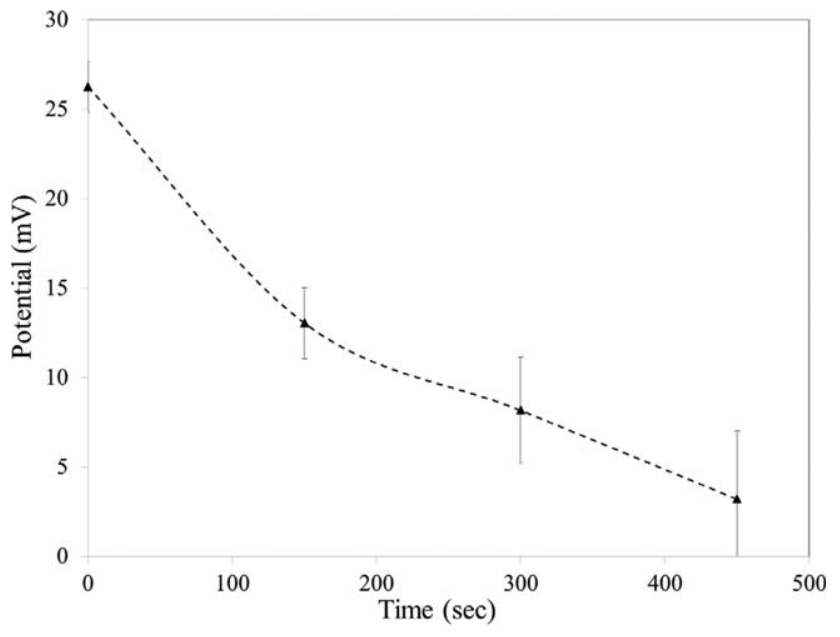

FIGURE 11 Loss of functionality of a receiver circuit as a function of exposure time to water. 
through modification of composition. The transiency mechanism of electronic components in this report is novel and different from the dissolution approach reported by others where the metals and semimetals like magnesium and silicon are chemically dissolved in the solvent (typically water) over a usually extended period of time. ${ }^{7,8,11,20}$ Here, the transiency of electronic components occurs very quickly and it is due to re-dispersion of silver microparticles in the solvent (water). This approach allows a two-stage transiency: a very quick initial transiency that disables the electronics and breaks components into micro-scale particles; and a second stage which allows chemical dissolution of the remains if they are soluble like magnesium. Silver microparticles used in this study are only for demonstration of electronic characteristics of bioelectronics and will not chemically dissolve in water, thus the stage two will never occur; not to mention silver and silver microparticles are not suitable metals to be implanted in the body especially for transient bioelectronics where the device is to be absorbed into the bioenvironments; however, silver-based electronics may be used for epidermal bioelectronics and other nonbiological applications such as military and environmental applications. For biological applications, biocompatible and biodegradable conductive pastes such as those based on tungsten and zinc can be used. $^{38}$ General findings and trends regarding straindependence of electronic properties are expected to be transformative to circuits fabricated based on other metal and semimetal nano and microparticles.

\section{CONCLUSIONS}

Circuits consisting of silver microparticle-based electronics were fabricated on PVA-based substrates and subjected to static and dynamic mechanical strain while their electronic properties were monitored and recorded. Conductivity of circuits was monitored and utilized as a means to deduce electronic properties when the circuits were subjected to different extents of mechanical strain at different frequencies. A correlation between electronic properties and strain was observed; yet, no correlation was evident between electronic properties and the frequency of the applied mechanical strain. It was also demonstrated that the composition of the polymeric substrate could be used as a means to control the transiency rate of bioelectronic circuits. This work is expected to contribute to design and fabrication of transient soft bioelectronics with metastable electronic properties and controlled transiency rate.

\section{EXPERIMENTAL}

\section{Materials}

Poly(vinyl alcohol) (PVA) (Mowiol 10-98, MW: 61,000 g $\mathrm{mol}^{-1}$, 98.0-98.8 mol \% hydrolysis), sucrose (98\%), hydrochloric acid, and acetone were purchased from Sigma-Aldrich (St. Louis, MO). Conductive silver ink (Pelco, 187) was purchased from Ted Pella.

\section{Membrane Casting}

$10 \mu \mathrm{l}$ of $1 \mathrm{M}$ hydrogen chloride ( $\mathrm{HCl}$ ) was added to $20 \mathrm{ml}$ of DI water and used as the base solvent. For each membrane composition, desired amount $(0,0.1,0.5$, and 2 gr $)$ of sucrose was dissolved in the base solvent, 1 gr of PVA was then added to the solution and stirred at $70{ }^{\circ} \mathrm{C}$ for $4 \mathrm{~h}$. The solution was then cooled down to ambient temperature and casted on a plastic mold (Teflon sheet for receiver substrate). Cured membranes were then carefully peeled off of the mold after 24 to $48 \mathrm{~h}$ depending on the composition.

\section{Electrically Conductive Patterns}

Conductive silver ink was diluted with acetone (1:1 volume ratio) and sprayed on the polymeric membranes over a vinyl mask of desired pattern with open area of $25 \mathrm{~mm}^{2}$. Vinyl masks with desired circuit designs were fabricated using a vinyl cutter (US-Cutter, SC series) with $25 \mu$ m planar resolution and $125 \mu \mathrm{m}$ repetition accuracy. Film thickness was controlled by monitoring the electric conductivity of the sprayed thin-films.

\section{Mechanical Characterization}

Mechanical characterization was performed on a Dynamic Mechanical Analyzer, (DMA-1, Mettler Toledo), loaded with tension clamp, at static and dynamic modes. Static testing was performed on force controlled mode for a range of 0-10 $\mathrm{N}$ at a rate of $0.5 \mathrm{~N} / \mathrm{min}$. Dynamic testing was performed under displacement controlled mode, at $1 \mathrm{~Hz}$ for fast oscillation and $0.2 \mathrm{~Hz}$ for slow oscillation; and, at $12 \mu \mathrm{m}$ for small displacement and $180 \mu \mathrm{m}$ for large displacement. All mechanical testing were isothermal. Clamps were isolated by paper-tape to prevent short circuit when electrical measurements were taken.

\section{Electrical Characterization}

Electronic properties of circuits under stress were monitored and recorded on a potentiostat (VersaSTAT 4, Princeton Applied Research) using VersaStudio software. Bias potential of $1 \mathrm{~V}$ was applied during the experiments and resistance was measured and recorded by the software. For stability control, a benchtop digital multimeter (DMM 4050, Tektronix) was used to directly monitor and measure resistance to five significant figures. All resistance data are normalized for the geometry of the sample.

\section{Degradation}

Transiency of circuits in DI water was monitored using a charged coupled device (CCD) camera. The extent of circuit degradation was deduced from loss of conductivity. Circuits were degraded in petri dish filled with DI water; external electrodes were isolated from contact with the solution to prevent current flow from the electrodes through the solution. Extra care was taken to ensure that current could only flow through the circuit on polymer substrate.

\section{Wirelessly Transmitted Signal}

An electric signal was generated using an induction coil (0.23 mm D wire, 755 turns, 6.0-8.0 $\Omega$ ) powered by a 17 $\mathrm{MHz}, 10 \mathrm{~V}_{\mathrm{pp}}$ square wave from an arbitrary function 
generator (AFG 3022B, Tektronix). The signal was received through a transient circuit connected to an oscilloscope (MSO2014, Tektronix). Experiments were conducted in faraday cage with shielded cables to reduce noise.

\section{Imaging}

A benchtop scanning electron microscopy (JCM-6000 NeoScope) was used to acquire high-resolution images of the silver electrodes and to confirm uniform coating. Close-up images of circuit were acquired using a digital microscope (AM7013MZT4, 5MP, Dino-Lite Premier); and other images were acquired using an ordinary CCD camera.

\section{ACKNOWLEDGMENTS}

This material is based upon work supported in part by the Department of Mechanical Engineering at Iowa State University; and a funding from Health Research Initiative and Presidential Initiative for Interdisciplinary Research at Iowa State University.

\section{REFERENCES}

1 X. Huang, Y. Liu, H. Cheng, W. J. Shin, J. A. Fan, Z. Liu, C. J. Lu, G. W. Kong, K. Chen, D. Patnaik, Adv. Funct. Mater. 2014, 24, 3846-3854.

2 L. Martiradonna, Nat. Mater. 2015, 14, 267-267

3 G. G. Malliaras, Biochim. Biophys. Acta 2013, 1830, 42864287.

4 C. Schmidt, Nature 2012, 483, S37-S37

5 M. Berggren, A. Richter-Dahlfors, Adv. Mater. 2007, 19, 32013213.

6 C. W. Park, S.-K. Kang, H. L. Hernandez, J. A. Kaitz, D. S. Wie, J. Shin, O. P. Lee, N. R. Sottos, J. S. Moore, J. A. Rogers, S. R. White, Adv. Mater. 2015, 27, 3783-3788.

7 S. W. Hwang, H. Tao, D. H. Kim, H. Cheng, J. K. Song, E. Rill, M. A. Brenckle, B. Panilaitis, S. M. Won, Y. S. Kim, Y. M. Song, K. J. Yu, A. Ameen, R. Li, Y. Su, M. Yang, D. L. Kaplan, M. R. Zakin, M. J. Slepian, Y. Huang, F. G. Omenetto, J. A. Rogers, Science 2012, 337, 1640-1644.

8 S. W. Hwang, D. H. Kim, H. Tao, T. Kim, S. Kim, K. J. Yu, B. Panilaitis, J. W. Jeong, J. K. Song, F. G. Omenetto, Adv. Funct. Mater. 2013, 23, 4087-4093.

9 H. L. Hernandez, S. K. Kang, O. P. Lee, S. W. Hwang, J. A. Kaitz, B. Inci, C. W. Park, S. Chung, N. R. Sottos, J. S. Moore, J. A. Rogers, S. R. White, Adv. Mater. 2014, 26, 7637-7642.

10 L. Yin, H. Cheng, S. Mao, R. Haasch, Y. Liu, X. Xie, S. W. Hwang, H. Jain, S. K. Kang, Y. Su, Adv. Funct. Mater. 2014, 24 645-658.

11 S. W. Hwang, J. K. Song, X. Huang, H. Cheng, S. K. Kang, B. H. Kim, J. H. Kim, S. Yu, Y. Huang, J. A. Rogers, Adv. Mater. 2014, 26, 3905-3911.

12 C. J. Bettinger, Z. Bao, Adv. Mater. 2010, 22, 651-655.

13 M. Muskovich, C. Bettinger, J. Adv. Healthcare Mater. 2012, 1, 248-266.
14 H. Acar, S. Cıınar, M. Thunga, M. R. Kessler, N. Hashemi, R. Montazami, Adv. Funct. Mater. 2014, 24, 4135-4143.

15 M. Irimia-Vladu, P. A. Troshin, M. Reisinger, L. Shmygleva, Y. Kanbur, G. Schwabegger, M. Bodea, R. Schwödiauer, A. Mumyatov, J. W. Fergus, Adv. Funct. Mater. 2010, 20, 40694076.

16 D. H. Kim, J. Viventi, J. J. Amsden, J. Xiao, L. Vigeland, Y. S. Kim, J. A. Blanco, B. Panilaitis, E. S. Frechette, D. Contreras, D. L. Kaplan, F. G. Omenetto, Y. Huang, K. C. Hwang, M. R. Zakin, B. Litt, J. A. Rogers, Nat. Mater. 2010, 9, 511-517.

17 C. Legnani, C. Vilani, V. Calil, H. Barud, W. Quirino, C. Achete, S. Ribeiro, M. Cremona, Thin Solid Films 2008, 517, 1016-1020.

18 M. Luo, A. W. Martinez, C. Song, F. Herrault, M. G. Allen, J. Microelectromech. Syst. 2014, 23, 4-13.

19 L. Yin, X. Huang, H. Xu, Y. Zhang, J. Lam, J. Cheng, J. A. Rogers, Adv. Mater. 2014, 26, 5.

20 S. W. Hwang, S. K. Kang, X. Huang, M. A. Brenckle, F. G. Omenetto, J. Rogers, Adv. Mater. 2015, 27, 47-52.

21 G. Lanzani, Nat. Mater. 2014, 13, 775-776.

$22 \mathrm{H}$. Ding, M. Zhong, Y. J. Kim, P. Pholpabu, A. Balasubramanian, C. M. Hui, H. He, H. Yang, K. Matyjaszewski, C. Bettinger, J. ACS Nano. 2014, 8, 4348-4357.

$23 \mathrm{~T}$. Goda, P. Kjall, K. Ishihara, A. Richter-Dahlfors, Y. Miyahara, Adv. Healthcare Mater. 2014, 3, 1693-1693.

24 A. A. Amiri Moghadam, W. Hong, A. Kouzani, A. Kaynak, R. Zamani, R. Montazami, Sens. Actuators A 2014, 217, 168-182.

25 W. Hong, A. Almomani, R. Montazami, Org. Electron. 2014, 15, 2982-2987.

26 W. Hong, C. Meis, J. R. Heflin, R. Montazami, Sens. Actuators B. 2014, 205, 371-376.

27 C. Meis, N. Hashemi, R. Montazami, J. Appl. Phys. 2014, 115, 134302

28 R. Montazami, D. Wang, J. R. Heflin, Int. J. Smart Nano Mater. 2012, 3, 204-213.

29 Y. Liu, R. Zhao, M. Ghaffari, J. Lin, S. Liu, H. Cebeci, R. G. de Villoria, R. Montazami, D. Wang, B. L. Wardle, J. R. Heflin, Q. M. Zhang, Sens. Actuators A. 2012, 181, 70-76.

30 R. Montazami, S. Liu, Y. Liu, D. Wang, O. Zhang, J. R. Heflin, J. Appl. Phys. 2011, 109, 104301

31 Y. Liu, S. Liu, J. Lin, D. Wang, V. Jain, R. Montazami, J. R. Heflin, J. Li, L. Madsen, Q. M. Zhang, Appl. Phys. Lett. 2010, 96, 223503

32 S. Liu, R. Montazami, Y. Liu, V. Jain, M. Lin, X. Zhou, J. R. Heflin, Q. M. Zhang, Sens. Actuators A. 2010, 157, 267-275.

33 S. Liu, R. Montazami, Y. Liu, V. Jain, M. Lin, J. R. Heflin, Q. Zhang, Appl. Phys. Lett. 2009, 95, 023505.

34 R. Zhang, Y. Chen, R. Montazami, Materials 2015, 8, 27352748.

35 J. Yang, S. Ghobadian, P. J. Goodrich, R. Montazami, N. Hashemi, Phys. Chem. Chem. Phys. 2013, 15, 14147-14161.

36 Z. Bai, J. Mendoza Reyes, R. Montazaml, N. Hashemi, J. Mater. Chem. A 2014, 2, 4878-4884.

37 J. D. Caplin, N. G. Granados, M. R. James, R. Montazami, N. Hashemi, Adv. Healthcare Mater. 2015, 4, 1426-1450. doi: 10.1002/adhm.201500040.

38 X. Huang, Y. Liu, S. W. Hwang, S. K. Kang, D. Patnaik, J. F. Cortes, J. A. Rogers, Adv. Mater. 2014, 26, 7371-7377. 